\title{
Permanent photodoping of plasmonic gallium-ZnO nanocrystals
}

\author{
Anzelms Zukuls, (D) ${ }^{a}$ Raivis Eglītis, ${ }^{a}$ Tanel Käämbre, ${ }^{a, b}$ Reinis Ignatans, ${ }^{c}$ \\ Krišjānis Šmits, ${ }^{d}$ Kristaps Rubenis, ${ }^{e}$ Dzintars Začs ${ }^{\dagger}$ and Andris Šutka (D) *a,b
}

Donor dopants in oxide semiconductors are compensated not only by valuable electrons but also by other point defects, leading to a decrease in electric conductivity and infrared absorption. We demonstrate that the electron compensation mechanism in $\mathrm{Ga}$ doped $\mathrm{ZnO}$ nanocrystals can be promoted by photodoping. Unexpectedly, the electrons from photodoping are stable in the open air for months.

\section{Introduction}

The possibility of tuning charge carrier concentrations in plasmonic semiconductor nanocrystals has attracted great atten- tion in both fundamental studies and applied research as it enables the adjustment of optical properties in the infrared region. These nanocrystals and materials can be used in smart windows, ${ }^{1}$ scintillators, ${ }^{2}$ sensors $^{3}$ and photovoltaic devices. ${ }^{4}$ Among various semiconductor oxide materials, $\mathrm{ZnO}$ has been widely used as a material with plasmonic properties and a host for $\mathrm{p}$ and n-type doping. ${ }^{5-8}$ This is due to its abundancy, low toxicity, wide band gap $(3.3 \mathrm{eV})$ and flexible crystal lattice, suitable for the incorporation of a large variety of dopants. ${ }^{9}$

${ }^{a}$ Research Laboratory of Functional Materials Technologies, Faculty of Materials Science and Applied Chemistry, Riga Technical University, Paula Valdena 3/7, LV-1048 Riga, Latvia.E-mail: andris.sutka@rtu.lv

${ }^{b}$ Institute of Physics, University of Tartu, Wilhelm Ostwald Str. 1, 50411 Tartu, Estonia

'Institute of Materials, École polytechnique fédérale de Lausanne, CH-1015 Lausanne, Switzerland

${ }^{d}$ Institute of Solid State Physics, University of Latvia, Kengaraga 8, LV-1063 Riga, Latvia

${ }^{e}$ Rudolfs Cimdins Riga Biomaterials Innovations and Development Centre of RTU, Institute of General Chemical Engineering, Faculty of Materials Science,

Riga Technical University, Pulka 3, Riga, LV-1007, Latvia

${ }^{f}$ Institute of Food Safety, Animal Health and Environment "BIOR", Lejupes iela 3, Riga, LV-1076, Latvia
To achieve plasmonic properties and infrared absorption, $\mathrm{ZnO}$ has been doped with $\mathrm{Ga}^{5-7} \mathrm{Al},{ }^{6,7} \mathrm{In}^{7}$ and Ge. ${ }^{8}$ Thereat the doping efficiency, i.e. achieving high enough dopant incorporation levels and electron concentration, presents the main challenge. To obtain plasmonic properties, the dopants have to replace the host cations in their lattice sites. ${ }^{10}$ Various synthesis and preparation approaches have been used to ensure efficient doping. ${ }^{6,10,11}$

Still, an effectively incorporated dopant does not guarantee the formation of a delocalized electron. For example, aliovalent donor dopants can be compensated (localised) by other point defects such as cation vacancies or oxygen interstitials. To obtain higher electron concentrations and stronger plasmonic absorption, the nanocrystals have been synthesized in more reductive or oxygen poor conditions. ${ }^{6,11}$

In the present study we show, that the plasmonic absorp- tion from itinerant electrons can be permanently increased by photodoping and that the added electrons remain stable even in ambient air. Photodoping is well reported in literature for various semiconductor materials including $\mathrm{TiO}_{2}{ }^{12}$ and $\mathrm{ZnO} .{ }^{13}$ The electrons accumulate in semiconductors when their nanocrystal colloids in hole scavenging medium (usually alcohols) are irradiated by light with energy exceeding the band gap value of the semiconductor. The holes are scavenged via photochemical oxidation of the alcohol while electrons populate the conduction band of the semiconductor. The populat-ing electrons significantly modulate the optical properties of the semiconductor due to plasmon resonance. ${ }^{14}$ However, upon exposure to air or other oxidants, the photogenerated electrons are normally removed from the conduction band of the semiconductor and the concentration of free charge car- riers will then return to its initial value.

Here we demonstrate stable photodoping of Ga doped $\mathrm{ZnO}$ (GZO) nanocrystals. The initial plasmonic absorption and concentration of delocalised electrons in GZO nanocrystals is low but is increased after photodoping. This can be attributed to a change in the compensation mechanism. The excess charge from the donor dopants in UV irradiated GZO nanocrystals is 
compensated by electrons, while the concentration of other point defects available for charge neutralisation is decreased.

\section{Results and discussion}

Materials were synthesized by solvothermal synthesis method as described in the Experimental section. The XRD analysis (Fig. 1(a)) shows that the obtained GZO nanocrystals have wurt- zite structure that corresponds to $\mathrm{ZnO}$ (JCPDS 36-1451) and belongs to the $P 63 m c$ space group with diffraction maxima at $2 \theta=31.8^{\circ}$ (100), $34.5^{\circ}(002), 36.3^{\circ}(101), 47.6^{\circ}(102), 56.6^{\circ}$

(110), 62.9 (103), 68.0 (112) and 69.1 (201). ${ }^{5}$ Rietveld analysis (Table 1) shows that Ga addition decreases the size of the crystallites in both (100) and (001) directions and introduces microstrain which is a good indication for Ga incorporation into the $\mathrm{ZnO}$ crystal lattice. The transmission electron microscopy (TEM) images of $\mathrm{ZnO}$ and $\mathrm{GZO}$ nanocrystals (Fig. 1

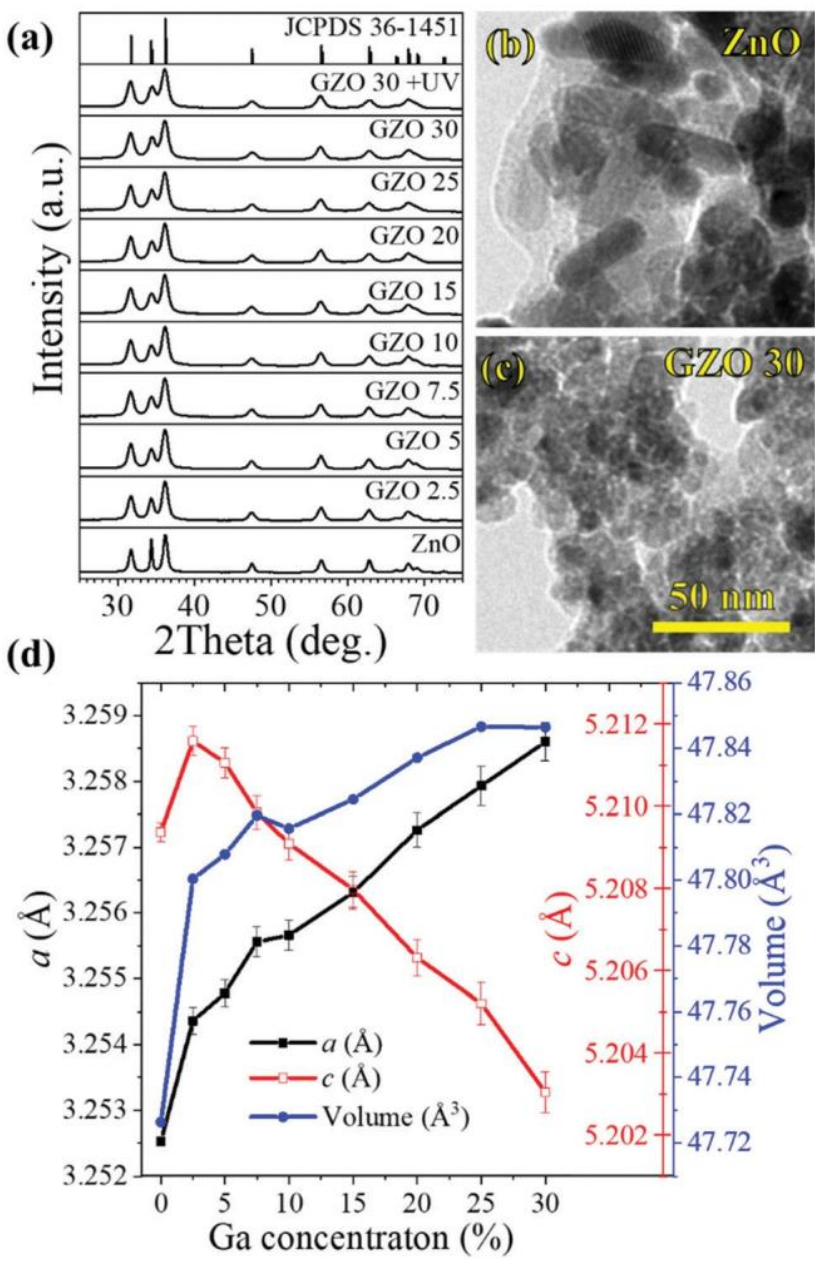

Fig. $1 \mathrm{X}$-ray diffraction patterns of the $\mathrm{ZnO}$ and GZO samples (a), TEM image for $\mathrm{ZnO}(\mathrm{b})$ and $\mathrm{GZO}$ with nominal $\mathrm{Ga} / \mathrm{Zn}$ content in the synthesis solution of $30 \%$ (c), the results of Rietveld refinement for lattice parameters and unit cell volume changes with $\mathrm{Ga}$ concentration in $\mathrm{ZnO}$ (d). The number in sample name on graphs (a), (b) and (c) indicate the nominal Ga concentration in $\mathrm{ZnO}$. and ESI Fig. S1†) confirm results from Rietveld analysis. The pristine $\mathrm{ZnO}$ nanocrystals have oblong shapes with diameters around $20 \mathrm{~nm}$ and length up to $50 \mathrm{~nm}$. The sizes of GZO nanocrystals in both directions are similar (around $20 \mathrm{~nm}$ ) regardless of the Ga doping level.

$\mathrm{Ga}$ addition increases the lattice parameter $a$, decreases the lattice parameter $c$ and expands the unit cell volume. Similar observations have been reported and related to combination of several factors such as smaller Ga four-fold coordination ionic radii $\left(\mathrm{Zn}^{2+}-0.74 \AA\right.$ and $\left.\mathrm{Ga}^{3+}-0.61 \AA\right),{ }^{15}$ relaxation of lattice parameters due to small crystallite sizes and the altered attractive forces between Ga dopants and oxygen neighbours along the $c$ axis.

Incorporation of $\mathrm{Ga}$ was also monitored by X-ray photoelectron spectroscopy (XPS). Fig. 2 shows fine scans over the $\mathrm{Ga} 2 \mathrm{p}$ region. The $\mathrm{Ga} 2 \mathrm{p}$ line shape is symmetric for all samples, which is the indication for single phase material for-mation. In combination with XRD studies that shows only a single crystalline phase, we conclude that $\mathrm{Ga}^{3+}$ is most prob- ably substituting $\mathrm{Zn}^{2+}$ in the $\mathrm{ZnO}$ lattice.

The changes in $\mathrm{Ga} 2 \mathrm{p}$ intensity relate to the $\mathrm{Ga} / \mathrm{Zn}$ ratio (all the corresponding $\mathrm{Zn} 2 \mathrm{p}$ were normalised to equal height). The amount of $\mathrm{Ga}$ in the $\mathrm{ZnO}$ increases by increasing the nominal $\mathrm{Ga}$ content into the synthesis solution (Table 1). The doping efficiency is higher at low nominal Ga concentrations. For example, the doping efficiency at nominal concentration of $2.5 \%$ reaches $80 \%$, while the doping efficiency at $30 \%$ nominal concentration is only $23 \%$ and the amount of $\mathrm{Ga}$ in the $\mathrm{ZnO}$ lattice reaches a mere $7 \%$. The additional XPS spectra (overview, $\mathrm{Zn} 2 \mathrm{p}$ and $\mathrm{O} 1 \mathrm{~s}$ ) are demonstrated in ESI Fig. S2-S4.†

The diffuse reflectance measurements (absorbance) for $\mathrm{ZnO}$ powders with different Ga content are demonstrated in Fig. S5.† All samples exhibit a sharp decrease in absorbance at around 360-370 $\mathrm{nm}$ that can be related to the absorption edge of $\mathrm{ZnO}$ band gap. The band gap values (Table S1†) of the syn- thesized materials vary slightly from $3.21 \mathrm{eV}$ to $3.13 \mathrm{eV}$ as observed from the optical absorption versus photon energy plots $(\alpha h \mathrm{v})^{2}$ (Fig. S6†). The infrared absorption that can be observed when incorporating $\mathrm{Ga}$ into $\mathrm{ZnO}$ can be assigned to the formation of delocalised electrons in the $\mathrm{ZnO}$ conduction band and corresponding plasmon resonance. ${ }^{5}$ Interestingly, regardless of the doping level, the infrared absorption of GZO samples is very similar, indicating that the excess dopants are compensated by other point defects such as Zn vacancies or oxygen interstitials.

For photodoping, the samples were dispersed in $n$-butanol and irradiated by UV light as described in the experimental section. After irradiation the samples were removed from the solvent, dried in air and used for diffuse reflectance measurements. As can be seen from Fig. 3 and Fig. S5, $†$ the light absorption in infrared range increases significantly for photodoped GZO samples. We observed also that the bandgap increases for the UV irradiated sample (Fig. 3(c)), which can be attributed to the Moss-Burstein shift. ${ }^{16}$ When the electronic states above the conduction band are populated by electrons, both absorption edge and the apparent bandgap are increased. 


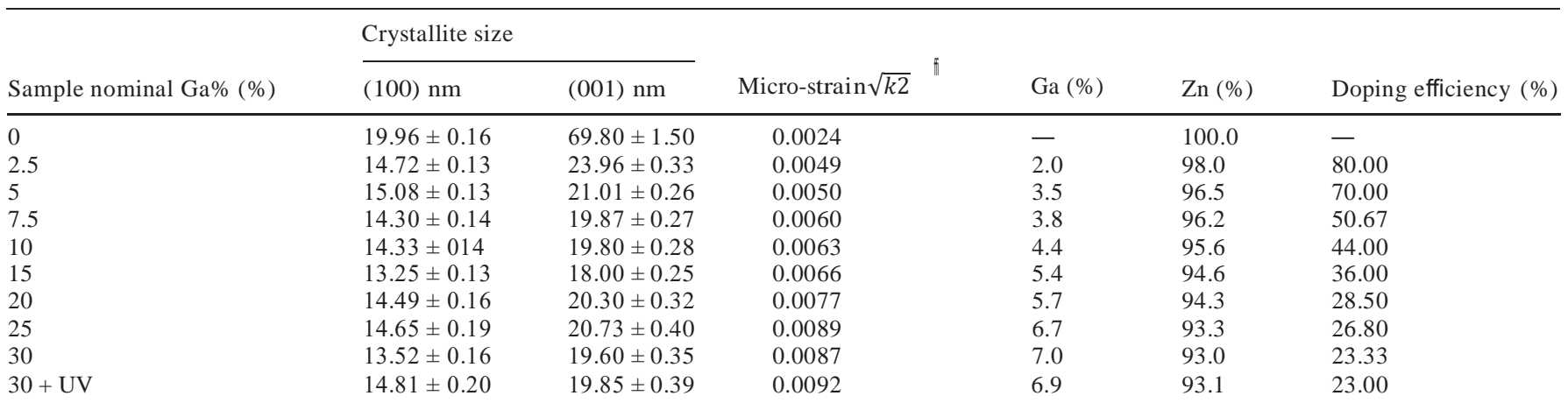

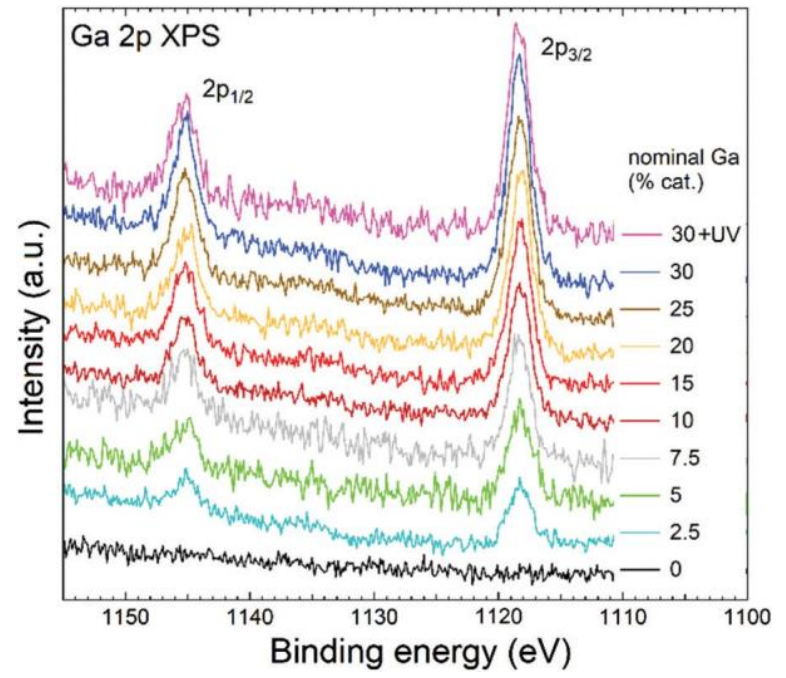

Fig. $2 \mathrm{Ga} 2 \mathrm{p}$ XPS spectra for different samples normalised to equal $\mathrm{Zn}$ $2 p$ height. The number in sample name indicate the nominal Ga concentration in $\mathrm{ZnO}$.
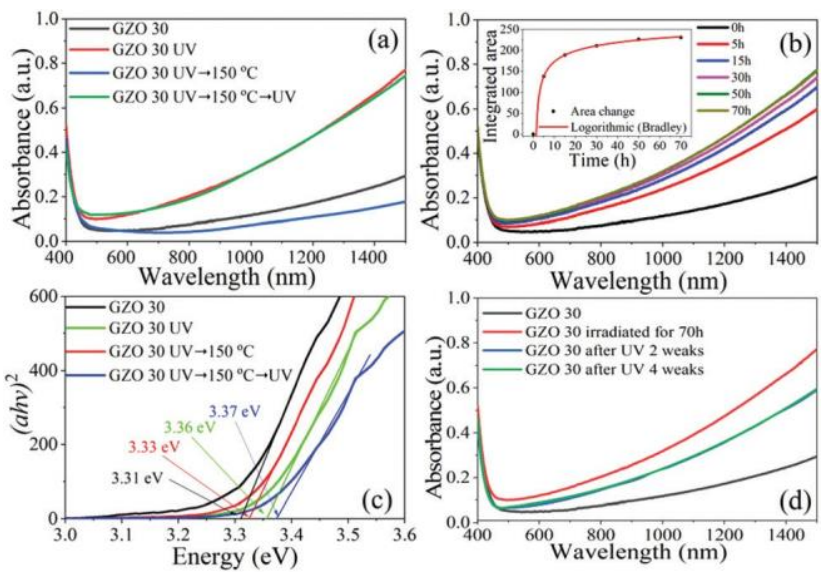

Fig. 3 UV-vis-NIR absorption spectra of GZO 30 powder: (a) before and after UV irradiation in different conditions; (b) the influence of photodoping duration on absorbance. Inset shows the integrated areas for the absorbance peaks in the 400-1500 nm range. Graph (c) shows optical band gaps for powder samples. Graph (d) shows the relaxation of infrared absorption after storing in air for 2 and 4 weeks.
The saturation in infrared absorption can be observed after 70 h of UV irradiation (Fig. 3(b) inset), but significant effect was observed even after 5 hours of irradiation. Optical measurements of the samples were repeated after they were stored in open vials for 2 and 4 weeks under ambient atmosphere conditions. As it can be seen in Fig. 3(d), the infrared absorption of the stored samples decreases slightly after 2 weeks, which can be attributed to oxidation. After this, the absorption remains unchanged for an additional 2 weeks at a significantly higher level than before the irradiation.

The observed phenomenon can't be related to improved crystallinity or changes in nanocrystal sizes, as well as the formation of additional point defects or activation of grain boundaries. As revealed by XRD (Fig. 1 and Fig. S7†) and Rietveld (Table 1) analysis, as well as TEM studies (Fig. S8†), no significant changes in crystal structures, grain boundaries or particle sizes were observed after photodoping. The perma- nent increase in the infrared absorption after photodoping can't be related also to changes in the doping level. We

studied the amount of $\mathrm{Ga}$ into a $\mathrm{ZnO}$ by XPS and did not observe any changes after photodoping (Table 1). Moreover, the $n$ - BuOH supernatant before UV irradiation was studied for the washed sample by inductively coupled plasma mass spectrometry (Table S2†). The amount of Ga dopant in the supernatant is $0.02 \%$. We thus conclude that the additional Ga doping if occurs, should not influence the infrared plasmonic absorption significantly. Further, we studied the defect structure of the GZO30 sample before and after UV photodoping. Following defect chemistry, the electron concentration in $\mathrm{ZnO}$ increases with an increase in the concentration of zinc interstitial and oxygen vacancies. No variations in spectral shape were observed for Zn 2p XPS signal (Fig. S2†). The O 1s XPS signal presented in Fig. S4† shows minor spectral changes. The O $1 \mathrm{~s}$ XPS signal (which was recorded using $\mathrm{Mg}-\mathrm{K}$ alpha excitation and thus does not suffer from the overlapping Zn Auger features visible in the overview spectra) was fitted with 3 components at $532.6 \mathrm{eV}, 531.7 \mathrm{eV}$ and $530.6 \mathrm{eV}$ of equal width, and with positions restricted to within $\pm 0.1 \mathrm{eV}$ between the fits of different samples. The component related to oxygen vacancies ${ }^{17-19}$ at $531.7 \mathrm{eV}$ show a minor relative increase from $25.96 \%$ to $26.23 \%$. The largest change from $12.20 \%$ to $17.55 \%$ 
is observed for the component at $532.6 \mathrm{eV}$ in the $\mathrm{O} 1 \mathrm{~s}$ XPS signal, which is related to chemisorbed oxygen species such as oxygen and $-\mathrm{OH}$ groups, ${ }^{17-19}$ which forms during the butanol oxidation by photogenerated holes. ${ }^{20}$

The plasmonic infrared absorption vanishes after annealing at $150{ }^{\circ} \mathrm{C}$ (Fig. 3). This can be attributed to oxygen incorporation and formation of oxygen interstitials that compensate additional charge from $\mathrm{Ga}^{3+}$ donor dopant, thus decreasing itinerant electron concentration. However, after re-dispersing in $n$ - $\mathrm{BuOH}$ and irradiating with UV, the permanent photodop- ing for annealed nanocrystals can be again observed at the same level (Fig. 3(a)). The increase in charge carrier concen- tration is indicated also by the increase of bandgap value due to the Moss-Burstein effect (Fig. 3(c)). The results clearly show that the permanent enhancement in infrared absorption comes from photodoping.

The observed phenomenon is not limited to GZO. Commercial Sn doped $\operatorname{In}_{2} \mathrm{O}_{3}$ (ITO) nanoparticles, $30 \mathrm{~nm}$ in size, also show a permanent increase in the visible and infrared absorption after photodoping (ESI Fig. S9†). The optical properties of photodoped ITO are preserving for at least four weeks. The permanent photodoping has not been observed for ITO nanoparticles before probably because the electron compensation mechanism already dominates into the synthesized materials. $^{21}$

The excess charge from $\mathrm{Ga}$ can be compensated by $\mathrm{Zn}$ vacancies, oxygen interstitials or electrons in accordance with eqn (1)-(3) below:

$$
\begin{gathered}
\mathrm{Ga}_{2} \mathrm{O}_{3} \stackrel{\mathrm{ZnO}}{\longrightarrow} 2 \mathrm{Ga}_{\mathrm{Zn}}^{\cdot}+3 \mathrm{O}_{\mathrm{O}}^{\times}+V^{\prime \prime}{ }_{\mathrm{zn}} \\
\mathrm{Ga}_{2} \mathrm{O}_{3} \stackrel{\mathrm{ZnO}}{\longrightarrow} 2 \mathrm{Ga}_{\mathrm{Zn}}^{\cdot}+2 \mathrm{O}_{\mathrm{O}}^{\times}+\mathrm{O}^{\prime \prime}{ }_{\mathrm{i}} \\
\mathrm{Ga}_{2} \mathrm{O}_{3} \stackrel{\mathrm{ZnO}}{\longrightarrow} 2 \mathrm{Ga}_{\mathrm{Zn}}^{\cdot}+2 \mathrm{O}_{\mathrm{O}}^{\times}+\frac{1}{2} \mathrm{O}_{2(\mathrm{~g})}+2 e^{-}
\end{gathered}
$$

The enhanced plasmon resonance infrared absorption is expected when process (3) dominates: the overall charge balance is maintained due to correspondingly high concentrations of delocalised electrons in the $\mathrm{ZnO}$ conduction band. ${ }^{5}$ During UV irradiation of NC suspensions in $n$-butanol the photogenerated holes are trapped while the photoexcited electrons remain in the conduction band. Under such circumstances, these delocalised electrons in conduction band can become the dominant stabilising factor in securing overall charge neutrality, and hence suppress the formation of other point defects (which has the same role of securing overall charge neutrality, but with the localisation of the electrons from the donor as a result).

The presence of defects and free electrons before and after GZO irradiation in the hole scavenging medium was studied also by photoluminescence (PL) measurements. The PL spectra for pristine and irradiated GZO samples are given in Fig. 4. The PL spectrum of pristine GZO show the narrow emis- sion peak at $380 \mathrm{~nm}$ arising from near band edge associated with free exciton recombination through an exciton-exciton collision process. ${ }^{22}$ Also, a broad blue emission band at

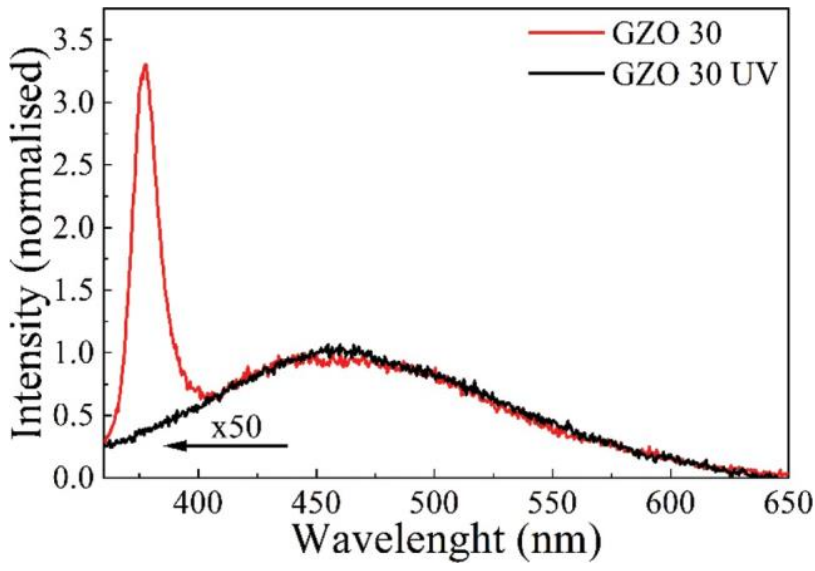

Fig. 4 Photoluminescence spectrum of GZO 30 sample before and after UV irradiation.

approximately $460 \mathrm{~nm}$ originating from oxygen vacancies and $\mathrm{Zn}$ interstitials is observed. ${ }^{23,24}$ After the UV irradiation, the PL from band edge and blue emission in GZO gets quenched due to enhanced Auger recombination - a clear indication of the presence of delocalized electrons in the semiconductor band gap. ${ }^{25}$ At the same time, there is no change in the spectral shape for PL blue emission at approximately $460 \mathrm{~nm}$ originat- ing from oxygen vacancies and $\mathrm{Zn}$ interstitials. This may indi- cate that there is no change in concentration of defects such as oxygen vacancies or zinc interstitials during the UV irradiation and all observed changes in infrared absorption for

UV irradiated GZO can be related for change of the delocalised electron concentration.

\section{Experimental}

Ga doped $\mathrm{ZnO}$ nanoparticles with different doping levels were solvothermally synthesized in methanol using zinc acetate dihydrate, gallium chloride and sodium hydroxide as precursors. First, $0.6 \mathrm{~g}$ of $\mathrm{NaOH}$ were dissolved in $30 \mathrm{~mL}$ of methanol by heating under stirring and reflux conditions in a two-neck round-bottom flask. Separately, $2 \mathrm{mmol}$ of $\mathrm{Zn}$ and Ga salts were dissolved in $15 \mathrm{ml}$ of methanol in desired ratio and injected into a $\mathrm{NaOH}$ solution. The $\mathrm{Ga} / \mathrm{Zn}$ nominal contents in solutions were $0,2.5,5,7.5,10,15,20,25$ and $30 \%$. After

injecting, the precipitates formed rapidly. The resulting mixture was boiled and stirred for 30 minutes. The suspen- sions were then moved to a Teflon lined pressure vessel and heated at 150 ${ }^{\circ} \mathrm{C}$ for 24 hours. Obtained samples were washed with methanol using centrifugation. Washed nanoparticles were dried at room temperature in air atmosphere. The mor- phology of the nanopowders was characterized using TEM (Tecnai G2 F20, FEI). The structure of the samples was studied by XRD (X'Pert PRO, PANalytical) using $\mathrm{Cu} \mathrm{Ka}$ radiation and XPS (Scienta SES100) hemispherical analyser operated at $200 \mathrm{eV}$ pass and using Al-Ka radiation, with an overall resolution of approximately $0.8 \mathrm{eV}$. The numerical percentage values of 
substitutional Ga were obtained using CasaXPS software, considering the photoionisation cross sections. Crystalline lattice parameters were determined from the measured XRD patterns by Rietveld refinement using Profex software. ${ }^{26}$ Sample irradiation in a hole scavenging medium ( photodoping) was done by using a $125 \mathrm{~W} 365 \mathrm{~nm}$ UV lamp. During the UV irradiation the constant room temperature was maintained by a fan ventilating system. Absorbance measurements were carried out using UV-VIS-NIR spectrophotometer (UV-3700, Shimadzu Scientific Instruments Kyoto, Japan).

ITO nanopowder (Sigma Aldrich, particle size $30 \mathrm{~nm}$ ) irradiation in a hole scavenging medium ( photodoping) was done by $254.7 \mathrm{~nm}$ UVC light in a sealed quartz cuvette. The obtained powder was separated from $n$-butanol using a centrifuge and dried.

Elemental analysis for supernatant was carried out with an inductively coupled plasma mass spectrometer (ICP-MS). Samples were diluted 1000 times with acidified water and analysed using the Agilent 7700x ICP-MS system (Agilent Technologies, Japan). Plasma was set at normal, robust mode, RF power was set at $1300 \mathrm{~W}$, while carrier gas and dilution gas flows were kept 0.6 and $0.4 \mathrm{~L} \mathrm{~min}^{-1}$, respectively.

Photoluminescence measurements were performed using a spectrograph (B-303i, Andor Shamrock) and sample luminescence excitation was done with YAG laser FQSS266 using 4th harmonic at $266 \mathrm{~nm}$ (slit size $50 \mu \mathrm{m}$ and filter - BS5).

\section{Conclusions}

GZO nanocrystals have been successfully synthesized in methanol by a solvothermal approach. Obtained nanocrystals with various Ga doping levels exhibit infrared surface plasmon resonance. The absorption can be significantly enhanced by photodoping - UV irradiation of nanocrystal dispersions in a hole scavenging medium ( $n$-butanol). Irradiated nanocrystals retained increased infrared absorption even after exposure to atmospheric oxygen. Observed permanent effect is related to electron compensation becoming the dominant compensation mechanism for $\mathrm{Ga}$ dopant charge as the result of the photodoping.

\section{Conflicts of interest}

There are no conflicts to declare.

\section{Acknowledgements}

This work was supported by the Latvian Council of Science in the framework of FLPP (Plasmonic oxide quantum dots for energy saving smart windows, lzp-2018/1-0187). Tanel Käämbre acknowledges financial support for the XPS instrumentation maintenance from the Estonian Centre of Excellence in Research project "Advanced materials and high- technology devices for sustainable energetics, sensorics and nanoelectronics" (TK141).

\section{Notes and references}

1 Z. Liu, Y. Zhong, I. Shafei, R. Borman, S. Jeong, J. Chen, Y. Losovyj, X. Gao, N. Li, Y. Du, E. Sarnello, T. Li, D. Su,

W. Ma and X. Ye, Nat. Commun., 2019, 10, 1-11.

2 E. D. Bourret-Courchesne, S. E. Derenzo and M. J. Weber, Nucl. Instrum. Methods Phys. Res., Sect. A, 2009, 601, 358363.

3 A. Agrawal, I. Kriegel and D. J. Milliron, J. Phys. Chem. C, 2015, 119,6227-6238.

4 H. Mahdhi, Z. Ben Ayadi, J. L. Gauffier, K. Djessas and

S. Alaya, J. Mater. Sci.: Mater. Electron., 2015, 26, 3336-3343.

5 M. Saha, S. Ghosh, V. D. Ashok and S. K. De, Phys. Chem. Chem. Phys., 2015, 17, 16067-16079.

6 M. K. Hamza Taha, O. Boisron, B. Canut, P. Melinon, J. Penuelas, M. Gendry and B. Masenelli, RSC Adv., 2017, 7, 28677-28683.

7 E. Della Gaspera, A. S. R. Chesman, J. Van Embden and J. J. Jasieniak, ACS Nano, 2014, 8, 9154-9163.

8 E. Della Gaspera, N. W. Duffy, J. Van Embden, L. Waddington, L. Bourgeois, J. J. Jasieniak and A. S. R. Chesman, Chem. Commun., 2015, 51, 1236912372.

9 P. Singh, R. Kumar and R. K. Singh, Ind. Eng. Chem. Res., 2019, 58, 17130-17163.

10 R. Buonsanti and D. J. Milliron, Chem. Mater., 2013, 25, 1305-1317.

11 A. Šutka, A. Zukuls, R. Eglītis, T. Käämbre, M. Kook, S. Vlassov, K. Rubenis and R. Ignatans, Phys. Status Solidi, 2019, 1900335, 1-5.

12 U. Joost, A. Šutka, M. Oja, K. Smits, N. Döbelin, A. Loot, M. Järvekülg, M. Hirsimäki, M. Valden and E. Nõmmiste, Chem. Mater., 2018, 30, 8968-8974.

13 A. M. Schimpf, C. E. Gunthardt, J. D. Rinehart, J. M. Mayer and D. R. Gamelin, J. Am. Chem. Soc., 2013, 135, 1656916577 .

14 A. Agrawal, S. H. Cho, O. Zandi, S. Ghosh, R. W. Johns and D. J. Milliron, Chem. Rev., 2018, 118, 3121-3207.

15 J. U. Brehm, M. Winterer and H. Hahn, J. Appl. Phys., 2006, 100, 1-9.

16 U. Joost, A. Šutka, M. Oja, K. Smits, N. Döbelin, A. Loot, M. Järvekülg, M. Hirsimäki, M. Valden and E. Nõmmiste, Chem. Mater., 2018, 30, 8968-8974.

17 F. Kayaci, S. Vempati, I. Donmez, N. Biyikli and T. Uyar, Nanoscale, 2014, 6, 10224-10234.

18 A. Kushwaha and M. Aslam, J. Phys. D: Appl. Phys., 2013, 46, 485104.

19 Y. Tu, S. Chen, X. Li, J. Gorbaciova, W. P. Gillin, S. Krause and J. Briscoe, J. Mater. Chem. C, 2018, 6, 1815-1821.

20 P. Kostestkyy, J. Yu, R. J. Gorte and G. Mpourmpakis, Catal. Sci. Technol., 2014, 4, 3861-3869. 
21 A. M. Schimpf, S. D. Lounis, E. L. Runnerstrom, D. J. Milliron and D. R. Gamelin, J. Am. Chem. Soc., 2015, 137, 518-524.

22 D. Chu, Y. Masuda, T. Ohji and K. Kato, Langmuir, 2010, 26, 2811-2815.

23 Q. Jiang, Z. Y. Wu, Y. M. Wang, Y. Cao, C. F. Zhou and J. H. Zhu, J. Mater. Chem., 2006, 16, 1536-1542.

24 L. Saikia, D. Bhuyan, M. Saikia, B. Malakar, D. K. Dutta and P. Sengupta, Appl. Catal., A, 2015, 490, 4249.

25 M. Shim and P. Guyot-Sionnest, J. Am. Chem. Soc., 2001, 123, 11651-11654.

26 N. Doebelin and R. Kleeberg, J. Appl. Crystallogr., 2015, 48, $1573-1580$.

Institute of Solid State Physics, University of Latvia as the Center of Excellence has received funding from the European Union's Horizon 2020 Framework Programme H2020-WIDESPREAD-01-2016-2017-TeamingPhase2 under grant agreement No. 739508, project CAM ART2 